\title{
1 Amino acid and nucleotide metabolism 2 shape the selection of trophic levels in 3 animals
}

4 Rosemary $\mathrm{Yu}^{1,2}$, Hao Wang ${ }^{1,3}$, Jens Nielsen ${ }^{1,2,4,5, *}$

${ }^{1}$ Department of Biology and Biological Engineering, Chalmers University of Technology, SE-412 96 Gothenburg, Sweden.

${ }^{2}$ Novo Nordisk Foundation Center for Biosustainability, Chalmers University of Technology, SE-412 96 Gothenburg, Sweden.

${ }^{3}$ National Bioinformatics Infrastructure Sweden, Science for Life Laboratory, Chalmers University of Technology, SE-412 58, Gothenburg, Sweden.

${ }^{4}$ Novo Nordisk Foundation Center for Biosustainability, Technical University of Denmark, DK-2800 Kgs. Lyngby, Denmark.

${ }^{5}$ Biolnnovation Institute, Ole Måløes Vej 3, DK-2200 Copenhagen N, Denmark.

\section{* Corresponding author:}

Jens Nielsen, nielseni@chalmers.se

\section{Abstract}

What an animal eats determines its trophic level (TL) in the food web. The diet of high-TL animals is thought to contain more energy because it contains higher levels of lipids. This however has not been systematically examined in the context of comprehensive metabolic networks of different animals. Here, we reconstruct species-specific genome-scale metabolic models (GEMs) of 32 animals, and calculate the maximum ATP production per unit of food for each animal. Surprisingly, we find that ATP production is closely associated with metabolic flux through central carbon metabolism and amino acid metabolism, while correlation with lipid metabolism is low. Further, metabolism of specific amino acids and nucleotides underlie maximum ATP production from food. Our analyses indicate that amino acid and nucleotide metabolism, rather than lipid metabolism, are major contributors to the selection of animal trophic levels, demonstrating that species-level metabolic flux plays key roles in trophic interactions and evolution. 


\section{Introduction}

The choice of food used by an organism for nutritional intake, and the breakdown of nutrients to generate energy in the form of adenosine triphosphate (ATP), are fundamental to living systems. Animals exhibit a wide diversity of dietary choices which place them at different trophic levels (TL) in a food chain or food web (Fig 1A). In terms of food availability, plants and algae/phytoplankton are exceptionally abundant and stable dietary resources, and have been so across evolutionary history ${ }^{1-}$

${ }^{3}$. Moreover, a plant-based diet is estimated to be $>10$-fold more ecologically efficient than an animal-based diet, since only a fraction of the energy available in the biomass of a given TL is transferred to the next upper $\mathrm{TL}^{4-7}$. Consistently, the evolutionary diversification rate of herbivores has been found to be faster than that of carnivores ${ }^{8}$, while specialization in carnivory appears to be unstable, as it is associated with short extinction times and repeated ecological replacements ${ }^{9,10}$. Surprisingly, however, recent large-scale studies have shown that a relatively small proportion of animal species are herbivores (32-43\%), whereas $57-66 \%$ of species have a diet consisting, partially or completely, of other animals ${ }^{8,11}$. This suggests a selective advantage in an animal-based diet, which thereby favors the selection of high-TL animals.

The traditional assumption (e.g. ref ${ }^{12}$ ) for this selection pressure is that an animal-based diet contains more lipids, whereas a plant-based diet contains less lipids but more carbohydrates (Fig 1BC). As the energy density of lipids is higher than carbohydrates ${ }^{13,14}$, this suggests that high-TL animals would be able to extract more energy per unit of food, consistent with the observation that carnivores spend less time feeding than similar-sized herbivores ${ }^{15}$. In other words, dietary lipid to carbohydrate ratio is thought to be the main determinant of the amount of energy obtained by an organism per unit of food. However, in living systems energy is extracted as ATP through a complex network of metabolic reactions, and the bottleneck(s) of ATP production in the context of the metabolic network of different animals is unknown. Moreover, there is substantial differences in dietary protein content with respect to TL (Fig 1B-C), which has an energy density comparable to that of carbohydrates, which can further contribute to ATP production. A closer examination of the metabolic determinants in trophic selection is therefore warranted.

A genome-scale metabolic model (GEM) is a constraint-based modeling framework wherein the metabolic network of an organism is represented mathematically ${ }^{16,17}$. Simulations using GEMs are based on the concept of flux balance analysis (FBA ${ }^{16}$, and can be used to calculate the production level of a metabolite with given constraints in the intake of nutrients. GEMs for microorganisms have been used for such simulations for many years ${ }^{18,19}$, and recently a unified Human-GEM, containing 
8,378 metabolites and 13,072 reactions, has also been published ${ }^{20}$, following $>15$ years of a concerted community effort.

Here, by using Human-GEM as a template ${ }^{20}$, we report the reconstruction of GEMs for 32 animals and their use for performing FBA simulations at a range of TLs. Imposing constraints on these GEMs based on the dietary composition of carbohydrates, lipids, and proteins allows the simulation of ATP production per unit of food in each species. Interestingly, simulation results show that ATP production in relation to TL is associated with dietary protein content, rather than dietary lipid content. We further show that TL is substantially correlated with metabolic flux through most reactions in central carbon metabolism and amino acid metabolism (in particular Lys, Trp, Tyr, lle, Leu, and Val), but only a small proportion of reactions in lipid metabolism. Finally, we find that metabolic pathways of specific amino acids (His metabolism, Thr-Gly conversion, and Asn-Asp conversion) and nucleotides underlie maximum ATP production from food. Taken together, our analyses indicate that amino acid and nucleotide metabolism play major roles in shaping the metabolism of high-TL animals, and suggest that species-level metabolic flux can play key roles in trophic interactions and evolution.

\section{Results}

\section{Dietary nutrient composition}

We reconstructed species-specific animal GEMs for a total of 32 species, including 22 terrestrial and 11 aquatic organisms, with TL ranging from 2 to 4.2 (Supplementary Table 1; see Methods section for data source and related calculations). To constrain the models based on dietary nutrient composition, we calculated the \% ( $\mathrm{g} / \mathrm{g}$ wet weight) of dietary carbohydrates, lipids, and proteins for each species (Supplementary Table 1), based on attributes mined from EltonTraits ${ }^{21}$ and FishBase ${ }^{22}$ (see Methods section). Consistent with previous literature, dietary carbohydrate content decreases with TL (Fig 2A). At around TL = 2, the dietary carbohydrate content varies between $2-8 \%$, as there are large differences in the carbohydrate content in different parts of plants (Fig 1B-C). The diet of the herbivorous $(\mathrm{TL}=2.05$ ) fish Oreochromis niloticus (Nile tilapia) contains $11 \%$ carbohydrates, reflecting the high carbohydrate content in algae ${ }^{23}$. Above around $\mathrm{TL}=3$, dietary carbohydrates of both terrestrial and aquatic species decreased below 1\% (Fig 2A). In contrast, the dietary content of proteins increases with TL, from $2 \%$ to $19 \%$, and data from both terrestrial and aquatic species follow closely the same trajectory (Fig 2B). The dietary content of lipids also increases with TL, however data from terrestrial and aquatic species clearly follow distinct trajectories, with terrestrial species reaching up to $10 \%$ dietary lipids at high $\mathrm{TL}$, while aquatic species reaching only $4 \%$ (Fig $2 \mathrm{C}$ ). The diet 
of Oreochromis niloticus contains $6 \%$ lipids, again reflecting the biomass composition of algae ${ }^{23}$, which places the dietary lipid content of this fish in the trajectory of terrestrial animals (Fig $2 \mathrm{C}$ ).

\section{ATP production is not associated with dietary lipid content}

We used FBA to simulate the maximum ATP production for each species, given the constraints in dietary carbohydrates, proteins, and lipids. The reactions of $\mathrm{O}_{2}$ uptake, $\mathrm{CO}_{2}$ production, as well as exchange reactions of $\mathrm{H}_{2} \mathrm{O}$, protons, and metal ions, were unconstrained. For nitrogenous waste, the exchange reactions of urea, urate, allantoin, or $\mathrm{NH}_{3}$, were either unconstrained or constrained to 0 , to reflect the species-specific waste product (see Methods section). All other exchange reactions were constrained to 0 . We found that ATP production increased with increasing TL (Fig 2D), and importantly, it is not grouped into distinct trajectories depending on the habitat of the animals, as is the case with dietary lipid content (Fig $2 \mathrm{C}$ ). This indicates that, contrary to the traditional assumption 12, lipid content is not the limiting dietary nutrient in ATP production in relation to TL.

To further examine the metabolic constraints of ATP production from the diet in different animals, we constrained the ATP production reaction in each GEM to the maximum calculated value (Fig 2D), and implemented FBA with random sampling ${ }^{24}$ to obtain a set of 1,000 possible flux distributions within the feasible region. The average flux of each reaction (Supplementary Table 2) was then correlated with $\mathrm{TL}$, and hereby we found that a large proportion of reactions in metabolic subsystems related to central carbon metabolism and amino acid metabolism are highly correlated with TL (Fig 2E). In contrast, the pathways related to lipid metabolism have relatively few reactions correlating with TL (Fig 2E), in agreement with the result that ATP production is not constrained by the dietary lipid content of animals at different TLs.

\section{Metabolic fluxes correlated with TL}

We then examined the specific reactions and pathways that are found to be highly correlated with TL. In the glycolysis-gluconeogenesis pathway, almost all reactions in 'lower' glycolysis, involving a chain of metabolic conversions between 3-carbon molecules, are highly correlated with TL (Fig 3A); whereas reactions in 'upper' glycolysis, involving the metabolism of 6-carbon molecules, are not. The reaction that connects upper and lower glycolysis (HMR_4375r), which converts the 6-carbon molecule fructose 1,6-bisphosphate (F1,6-bP) to 3-carbon molecules dehydroxyacetone phosphate (DHAP) and glyceraldehyde 3-phosphate (G3P), shows borderline correlation with $\mathrm{TL}$ with $\rho_{\text {spearman }}=$ 0.75 (Fig 3A). In particular, lower glycolysis generally carries positive flux in low-TL species, consistent with the use of glycolysis to metabolize the high levels of carbohydrates in the diet of these organisms (Fig 2A). In high-TL species, this pathway carries negative flux (Fig 3A), indicating the use of gluconeogenesis to synthesize other metabolic intermediates, at a cost of ATP. 
In the tricarboxylic acid (TCA) cycle, we found that the first step of the pathway (HMR_4145r) catalyzing the entry of acetyl-CoA into the cycle, as well as several steps in the second half of the cycle converting succinyl-CoA (Suc-CoA) to malate (MAL), are highly correlated with TL (Fig 3B). This is likely related to high levels of acetyl-CoA and Suc-CoA entering the TCA cycle in high-TL species. As the high dietary protein levels (Fig 2B) provides disproportionate levels of amino acids, many of the amino acids are deaminated and converted into acetyl-CoA or succinyl-CoA, entering the TCA cycle to generate ATP. As an example, Fig 3 C shows the degradation of lysine to acetyl-CoA, wherein several steps are highly correlated with TL. The degradation of aromatic amino acids to acetyl-CoA, and branched chain amino acids to succinyl-CoA, all follow similar trends (Supplementary Table 2).

\section{ATP production is constrained by nucleotide metabolism}

In amino acid metabolic pathways, beyond the increase in the degradation of amino acids to acetylCoA and Suc-CoA within increasing TL (Fig 3B-C and Supplementary Table 2), we also found that TL is highly correlated with several amino acids being shunted towards the synthesis of metabolic intermediates in nucleotide synthesis. In particular, nearly all steps in the conversion of histidine to 10-formyl THF, which enters the nucleotide metabolic pathway at two distinct steps, are highly correlated with TL, with an overall $\rho_{\text {Spearman }}=0.96$ (Fig 4A). The conversion of threonine to glycine, and asparagine to aspartate, are similarly highly correlated with $\mathrm{TL}\left(\rho_{\text {Spearman }}=0.94\right.$ and 0.85 respectively), consistent with an increased supply of GAR (Glycineamideribotide) and SAICAR (Phosphoribosylaminoimidazolesuccinocarboxamide) to the nucleotide metabolic pathway (Fig 4A). Moreover, reactions in the entire pathway of IMP (inosine monophosphate) production from PRPP (phosphoribosyl pyrophosphate), all exhibit high correlations with $\operatorname{TL}\left(\rho_{\text {spearman }}=0.96\right)$, suggesting that nucleotide metabolism, rather than the lipid metabolism, plays a significant role in ATP production in animals at different TLs.

While it makes intuitive sense that ATP production is constrained by the metabolic pathway catalyzing the synthesis of nucleotides, these results do not account for any nucleotides that are already available as a part of the diet (free or bound in DNA/RNA). As measurements of nucleotide content in different dietary sources are scarce, we addressed this by constraining our model to allow a dietary AMP intake of up to $10 \%$ (g/g wet weight). We then used GEMs to simulate the maximum ATP production in each species as before. Our results show that allowing for up to $10 \%$ of dietary AMP led to an up-shift in the maximum ATP production in each species by $\sim 29 \mathrm{mmol}$, but did not alter the overall trajectory of ATP production with respect to TL (Fig 4B). Data from terrestrial and aquatic species remained on the same trajectory, instead of separating into distinct groups which would be the case if dietary lipids were the constraining nutrient for ATP production (Fig 2C). We 
therefore conclude that amino acid and nucleotide metabolism play key roles in the production of ATP from food, which in turn contributes to the evolutionary selection of animals at high TL.

\section{Discussion}

We reconstructed species-specific GEMs for 32 different animals and simulated the maximum ATP production per unit of food. Our analyses show that dietary protein content, rather than dietary lipid content, supports an increased ATP production that correlates with increasing TL. Results further indicate that ATP production from food is limited by the metabolic pathways of histidine metabolism, threonine-glycine conversion, asparagine-aspartate conversion, and nucleotide metabolism. We therefore conclude that amino acid and nucleotide metabolism are major contributors to the evolutionary selection of animal-based diets and high-TL animals, contrary to the traditional assumption ${ }^{12}$ of lipid content being the primary selection pressure.

Despite the prevalent use of dietary lipid content to explain the selection of high-TL animals, this does not always fit existing data. For instance, while animal-based diets are generally thought to contain higher levels of both proteins and lipids compared to plant-based diets, this is only true of terrestrial environments. In aquatic environments, the lipid content of fish is known to be very low, whereas the biomass composition of algae contains higher levels of lipids ${ }^{23}$ than fish ${ }^{22,25}$ or aquatic invertebrates ${ }^{26}$. As such, in aquatic environments, the diet of herbivorous species actually contains more lipids than omnivorous or carnivorous species. Therefore, dietary lipid content cannot explain the selection of high-TL species in aquatic ecosystems, suggesting that alternative factors are involved. Of the three main energy-carrying macronutrients in the diet, only the protein content is higher in omnivorous/carnivorous fish than in herbivorous fish (here represented by Oreochromis niloticus, Fig $2 \mathrm{~A}-\mathrm{C}$ ), suggesting that dietary protein content plays a key role in the selection of high-TL species, in line with our GEM simulation results.

Our results also show that, when a dietary nucleotide content of $10 \%$ is included as a simulation constraint upper bound, this leads to an up-shift in the maximum ATP production in all species, by an equal amount of $\sim 29 \mathrm{mmol}$ (Fig 4B). In reality, however, this up-shift is likely not constant across all species, but rather increases with increasing TL. This is because nucleotide levels generally track with protein levels, in part because the majority of RNA in living cells is ribosomal RNA which is closely associated with ribosomal proteins ${ }^{27}$. Thus, diets of high-TL animals would contain higher levels of both proteins and nucleotides, leading to a steeper slope of ATP production with respect to TL. Moreover, in low-TL animals, plant-based diets could contain toxins or anti-nutrients which limit the bioavailability of nutrients ${ }^{28,29}$. For example, trypsin inhibitors and hemagglutinins found in legumes can reduce the digestibility of proteins and amino acids by up to $50 \%$; tannins found in cereals have 
similar effects by up to $23 \%$; phytates in oilseeds, by up to $10 \%$; and many more ${ }^{29}$. These factors would further increase the steepness of the slope of ATP production with respect to TL, providing additional selection pressure for high-TL animals.

In addition to amino acid and nucleotide metabolism, our analyses show that metabolic flux through lower glycolysis and the second half of the TCA cycle, are also correlated with TL. Of particular note is that with increasing TL there is a reversal of the direction of flux in lower glycolysis, catalyzing glycolysis in low-TL animals, and gluconeogenesis in high-TL animals (Fig 3A). However, enzymes in this pathway are highly conserved across all organisms ${ }^{30,31}$, suggesting that the versatile use of this pathway to metabolize dietary nutrients in both directions is independent of enzyme properties and likely reflects the biochemistry of the pathway itself. Indeed, recently it has been shown that, out of hundreds of (theoretically) feasible alternative biochemical paths connecting G3P to pyruvate, the extant lower glycolysis is the optimal solution which carries the maximum flux for both glycolysis and gluconeogenesis under biologically relevant conditions ${ }^{32}$. For all other pathways found to correlate with TL, in particular the amino acid and nucleotide metabolism pathways involved in maximizing ATP production, whether adaptations in enzyme properties or pathway optimality underlies the selection of animal trophic levels remains an interesting open question.

\section{Methods}

\section{GEM reconstruction}

The animal GEMs were generated via an orthology-based approach, by using the RAVEN 2.0 package ${ }^{33}$ and the Human-GEM version $1.7^{20}$ as a template. The annotated orthologs and paralogs associated from human to other animal species were retrieved from the Ensembl database version $103^{34}$.

\section{Diet type and TL calculations}

Diets of terrestrial animals and whales (Delphinapterus leucas and Physeter catodon; see Supplementary Table 1) are obtained from EltonTraits ${ }^{21}$ which contains the percent usage of 10 diet types. The diet type "Inv" (invertebrates) is further split to differentiate the usage of aquatic invertebrates or terrestrial invertebrates, based on "Food Habits" data mined from Animal Diversity $\mathrm{Web}^{35}$, to a total of 11 diet types. Diet types are mined at the genus level, to account for missing data in Animal Diversity Web. For genus with food habits of insects, terrestrial non-insect anthropoids, or terrestrial worms, terrestrial invertebrate usage is equal to Diet-Inv. For genus with food habits of aquatic or marine worms, aquatic crustaceans, echinoderms Cnidarians, other marine invertebrates, or zooplankton, aquatic invertebrate usage is equal to Diet-Inv. For the food habit of mollusks, aquatic invertebrate usage is equal to Diet-Inv only for genus with food mollusks of both 
mollusks and fish, in order to exclude species that eat snails. If a genus is found to eat both aquatic and terrestrial invertebrates, usage of Diet-Inv is split in half into terrestrial and aquatic invertebrate usage. If no data is available, Diet-Inv is assumed to be terrestrial invertebrate usage. The $T L_{j}$ of each diet type $j$ is then taken as follows: fruit, nectar, seed, and plant, $T L_{j}=1$; terrestrial invertebrates, $T L_{j}$ $=2$; vertebrate endoderms, vertebrate ectoderms, and fish, $T L_{j}=2.5$. The $T L_{i}$ of each species $i$ is then calculated as follows ${ }^{36}$ :

$T L_{i}=1+\sum_{j}\left(T L_{j} \cdot D C_{i j}\right)$

where $D C_{i j}$ represents the fraction of $j$ in the diet of $i^{21}$.

For aquatic species except whales (Delphinapterus leucas and Physeter catodon), $T L_{i}$ is obtained from FishBase ${ }^{22}$. The $T L_{j}$ of each diet type $j$ is taken as follows: algae, $T L_{j}=1$; aquatic invertebrates, $T L_{j}=2$; fish, $T L_{j}=3$ for species at $T L_{i}$ between 3 and 4 , and $T L_{j}=4$ for species at $T L_{i}$ between 4 and 5 . The fraction of $j$ in the diet of $i\left(D C_{i j}\right)$ is then calculated as above ${ }^{36}$.

\section{Dietary nutrient composition}

The dietary composition of carbohydrates, lipids, and proteins for the 11 diet types (see diet type and TL calculations section) are mined from existing knowledge bases as follows: fruit ${ }^{26}$; nectar ${ }^{37,38}$; seed, ${ }^{26}$; plant, ${ }^{26}$; algae, ${ }^{23}$; aquatic invertebrates, ${ }^{26}$; terrestrial invertebrates, ${ }^{39,40}$; vertebrate endoderms, ${ }^{21,26}$; vertebrate ectoderm, ${ }^{26,41}$; vertebrate fish, ${ }^{22,25}$. Vertebrate general/unknown and scavenge are taken as the average of vertebrate endoderm, ectoderm, and fish.

\section{FBA and random sampling}

Constraints of each GEM are imposed as follows: for dietary carbohydrates, lipids, and proteins (CLP), both the upper bound ( $\mathrm{ub}$ ) and the lower bound (Ib) of the glucose uptake reaction, lipid pool uptake reaction, and protein pool uptake reaction were constrained to the $\%(g / g) C L P$ in the diet (Supplementary Table 1), after conversion to $\mathrm{mmol}$ by the previously estimated molecular weight of the pool metabolites ${ }^{20}$. For $\mathrm{O}_{2}$ uptake, the ub is constrained to 0 , and the $\mathrm{lb}$ is constrained to -Inf (negative infinity). For $\mathrm{CO}_{2}$ production, the $\mathrm{ub}$ is constrained to $\mathrm{Inf}$, and the $\mathrm{Ib}$ is constrained to 0 . Nitrogenous waste production are as follows: Simian primates (including humans) ${ }^{42}$ excrete urate and urea; non-Simian mammals excrete urea and allantoin; and fish excrete urea and $\mathrm{NH}_{3}$. For each species, then, the allowed nitrogenous waste is constrained with ub to Inf and Ib to 0 . For water, proton, and metal ions (zinc, selenate, sulfate, sodium, magnesium, lithium, potassium, iodide, ferrous ion, ferric ion, cupric ion, phosphate, chloride, and calcium), ub is constrained to Inf and Ib is 
constrained to -Inf. ATP production is constrained with $\mathrm{lb}$ to 0 and $\mathrm{ub}$ to Inf. All other exchange reactions are constrained to 0 .

MATLAB R2019b (MathWorks, Inc., Natick, MA) with Gurobi solver (Gurobi Optimizer, Beaverton, OR) in the COBRA toolbox ${ }^{43}$ was used for all GEM simulations. In FBA, ATP production is set to be the objective function, and $\operatorname{lnf}$ (or -Inf) are converted to 1000 (or -1000) to avoid loops. In FBA with random sampling, ATP production is constrained with both $\mathrm{lb}$ and $\mathrm{ub}$ equal to the maximum ATP production calculated in the first iteration of FBA, followed by 1000 random samplings of a pair of randomly weighted objective functions, as implemented in the RAVEN toolbox ${ }^{33}$. Spearman correlation ( $\left.\rho_{\text {Spearman }}\right)$ between the mean of the 1000 random sampling results for each reaction in each species, and the TL of the species, is then calculated. Reaction flux is considered highly correlated with TL if $\rho_{\text {Spearman }}>0.75$ or $\rho_{\text {spearman }}<-0.75$, and if the number of species where the reaction carries non-0 flux is $>17$ (i.e. $50 \%$ of all species considered). In subsystem analysis, subsystems wherein $>2$ reactions are highly correlated with TL are considered.

\section{Data availability}

Diet and TL data on the species studied are in Supplementary Table 1. Processed simulation results are in Supplementary Table 2. Species-specific GEMs and related data are available in the GitHub repository at https://github.com/SysBioChalmers/GEMsforTrophicLevels.

\section{Code availability}

Custom scripts are available in the GitHub repository at

https://github.com/SysBioChalmers/GEMsforTrophicLevels.

\section{References}

1 Coates, M. I., Marcello Ruta \& Friedman, M. Ever Since Owen: Changing Perspectives on the Early Evolution of Tetrapods. Annual Review of Ecology, Evolution, and Systematics 39, 571592, doi:10.1146/annurev.ecolsys.38.091206.095546 (2008).

2 Nelsen, M. P., DiMichele, W. A., Peters, S. E. \& Boyce, C. K. Delayed fungal evolution did not cause the Paleozoic peak in coal production. Proc Natl Acad Sci U S A 113, 2442-2447, doi:10.1073/pnas.1517943113 (2016).

3 Frederick, E. The world's first animal was probably a carnivore. News from Science, doi:10.1126/science.aaz3304 (2019).

4 Lindeman, R. L. The Trophic-Dynamic Aspect of Ecology. Ecology 23, 399-418, doi:10.2307/1930126 (1942).

5 Kent, M. Advanced Biology. (OUP Oxford, 2000).

6 Spellman, F. R. The Science of Water: Concepts and Applications, Second Edition. (Taylor \& Francis, 2000).

7 Eshel, G. \& Martin, P. A. Diet, energy, and global warming. Earth interactions 10, 1-17 (2006). 
Price, S. A., Hopkins, S. S., Smith, K. K. \& Roth, V. L. Tempo of trophic evolution and its impact on mammalian diversification. Proc Natl Acad Sci U S A 109, 7008-7012, doi:10.1073/pnas.1117133109 (2012).

9 Van Valkenburgh, B., Wang, X. \& Damuth, J. Cope's rule, hypercarnivory, and extinction in North American canids. Science 306, 101-104, doi:10.1126/science.1102417 (2004).

10 Van Valkenburgh, B. Major patterns in the history of carnivorous mammals. Annual Review of Earth and Planetary Sciences 27, 463-493 (1999).

11 Roman-Palacios, C., Scholl, J. P. \& Wiens, J. J. Evolution of diet across the animal tree of life. Evol Lett 3, 339-347, doi:10.1002/evl3.127 (2019).

12 Stephens, D. W. \& Krebs, J. R. Foraging Theory. Vol. 1 (Princeton University Press, 1986).

13 Ben-Dor, M., Sirtoli, R. \& Barkai, R. The evolution of the human trophic level during the Pleistocene. Am J Phys Anthropol, doi:10.1002/ajpa.24247 (2021).

14 Hall, K. D. et al. Calorie for Calorie, Dietary Fat Restriction Results in More Body Fat Loss than Carbohydrate Restriction in People with Obesity. Cell Metab 22, 427-436, doi:10.1016/j.cmet.2015.07.021 (2015).

15 Shipman, P. \& Walker, A. The costs of becoming a predator. Journal of Human Evolution 18, 373-392 (1989).

16 Nielsen, J. Systems Biology of Metabolism. Annu Rev Biochem 86, 245-275, doi:10.1146/annurev-biochem-061516-044757 (2017).

17 Fang, X., Lloyd, C. J. \& Palsson, B. O. Reconstructing organisms in silico: genome-scale models and their emerging applications. Nat Rev Microbiol, doi:10.1038/s41579-020-00440-4 (2020). Edwards, J. S. \& Palsson, B. O. The Escherichia coli MG1655 in silico metabolic genotype: its definition, characteristics, and capabilities. Proc Natl Acad Sci U S A 97, 5528-5533, doi:10.1073/pnas.97.10.5528 (2000).

19 Forster, J., Famili, I., Fu, P., Palsson, B. O. \& Nielsen, J. Genome-scale reconstruction of the Saccharomyces cerevisiae metabolic network. Genome Res 13, 244-253, doi:10.1101/gr.234503 (2003).

20 Robinson, J. L. et al. An atlas of human metabolism. Sci Signal 13, doi:10.1126/scisignal.aaz1482 (2020).

21 Wilman, H. et al. EltonTraits 1.0: Species-level foraging attributes of the world's birds and mammals: Ecological Archives E095-178. Ecology 95, 2027-2027 (2014).

22 Froese, R. \& Pauly, D. FishBase (online). <www.fishbase.org $>$ (2021).

23 Boyle, N. R. \& Morgan, J. A. Flux balance analysis of primary metabolism in Chlamydomonas reinhardtii. BMC Syst Biol 3, 4, doi:10.1186/1752-0509-3-4 (2009).

24 Bordel, S., Agren, R. \& Nielsen, J. Sampling the solution space in genome-scale metabolic networks reveals transcriptional regulation in key enzymes. PLoS Comput Biol 6, e1000859, doi:10.1371/journal.pcbi.1000859 (2010).

25 Vaitla, B. et al. Predicting nutrient content of ray-finned fishes using phylogenetic information. Nat Commun 9, 3742, doi:10.1038/s41467-018-06199-w (2018).

26 Food Surveys Research Group. Food and Nutrient Database for Dietary Studies (online). $<$ https://www.ars.usda.gov/northeast-area/beltsville-md-bhnrc/beltsville-human-nutritionresearch-center/food-surveys-research-group/docs/fndds-download-databases/> (2021).

27 Lodish, H. F. et al. Molecular Cell Biology. (W.H. Freeman, 2000).

28 Birk, Y. The Bowman-Birk inhibitor. Trypsin- and chymotrypsin-inhibitor from soybeans. Int J Pept Protein Res 25, 113-131, doi:10.1111/j.1399-3011.1985.tb02155.x (1985).

29 Gilani, G. S., Cockell, K. A. \& Sepehr, E. Effects of antinutritional factors on protein digestibility and amino acid availability in foods. J AOAC Int 88, 967-987 (2005).

30 Ronimus, R. S. \& Morgan, H. W. Distribution and phylogenies of enzymes of the EmbdenMeyerhof-Parnas pathway from archaea and hyperthermophilic bacteria support a gluconeogenic origin of metabolism. Archaea 1, 199-221, doi:10.1155/2003/162593 (2003). 
34131 Verhees, C. H. et al. The unique features of glycolytic pathways in Archaea. Biochem J 375, 231-246, doi:10.1042/BJ20021472 (2003).

32 Court, S. J., Waclaw, B. \& Allen, R. J. Lower glycolysis carries a higher flux than any biochemically possible alternative. Nat Commun 6, 8427, doi:10.1038/ncomms9427 (2015). Wang, H. et al. RAVEN 2.0: A versatile toolbox for metabolic network reconstruction and a case study on Streptomyces coelicolor. PLoS Comput Biol 14, e1006541, doi:10.1371/journal.pcbi.1006541 (2018).

34 Yates, A. D. et al. Ensembl 2020. Nucleic Acids Res 48, D682-D688, doi:10.1093/nar/gkz966 (2020).

35 Myers, P. et al. The Animal Diversity Web (online). , <https://animaldiversity.org.> (2021).

36 Pauly, D. \& Palomares, M.-L. Fishing down marine food web: it is far more pervasive than we thought. Bulletin of marine science 76, 197-212 (2005).

37 Nicolson, S. W., Nepi, M. \& Pacini, E. Nectaries and Nectar. (Springer Netherlands, 2007).

38 Gottsberger, G., Schrauwen, J. \& Linskens, H. Amino acids and sugars in nectar, and their putative evolutionary significance. Plant Systematics and Evolution 145, 55-77 (1984).

39 Finke, M. D. Complete nutrient content of four species of feeder insects. Zoo Biol 32, 27-36, doi:10.1002/zoo.21012 (2013).

40 Kouřimská, L. \& Adámková, A. Nutritional and sensory quality of edible insects. NFS journal 4, 22-26 (2016).

41 Onadeko, A., Egonmwan, R. \& Saliu, J. Edible amphibian species: local knowledge of their consumption in southwest Nigeria and their nutritional value. West African journal of applied ecology 19 (2011).

42 Schoch, C. L. et al. NCBI Taxonomy: a comprehensive update on curation, resources and tools. Database (Oxford) 2020, doi:10.1093/database/baaa062 (2020).

43 Heirendt, L. et al. Creation and analysis of biochemical constraint-based models using the COBRA Toolbox v.3.0. Nat Protoc 14, 639-702, doi:10.1038/s41596-018-0098-2 (2019).

\section{Acknowledgements}

This research was supported by funding from the Novo Nordisk Foundation (grant number

NNF10CC1016517) and the Knut and Alice Wallenberg Foundation. Open access funding is provided

by Chalmers University of Technology.

373 R.Y., H.W., and J.N. conceived the study. R.Y. and H.W. designed and performed the analyses. J.N.

374 supervised the study. All authors wrote the manuscript.

376 The authors declare no competing interests related to this work.

\section{Materials and Correspondence}

378 Correspondence to Jens Nielsen (email: nielsenj@chalmers.se) 


\section{Supplementary information}

Supplementary Table 1-2.

\section{Figure legends}

382

Figure 1. Animal trophic levels (TL) and nutrient composition of different diet types. $\mathbf{A}$, two examples of food chains are shown to demonstrate the concept of TL. B-C, the composition of the three major dietary macronutrients (lipids, carbohydrates, and proteins) in different diet types of terrestrial (B) and aquatic (C) species, order by trophic level, is given in \% (g/g wet weight). Diet types are as collated in EltonTraits (Wilman et al, 2014).

Figure 2. Dietary nutrient composition and maximum ATP production. A-C, dietary carbohydrates (A), proteins (B), and lipids (C) of 33 species (32 animals plus human), is given with respect to the speciesspecific TL. Terrestrial species are in orange and aquatic species are in blue. $\mathbf{D}$, the maximum ATP production as simulated using species-specific GEMs and constrained to reflect $100 \mathrm{~g}$ of food intake. E, the metabolic subsystems wherein a high \% of reactions show high correlation with TL in FBA and random sampling analyses. Subsystems related to carbohydrate metabolism, amino acid metabolism, and fatty acid metabolism are shown.

Figure 3. Select metabolic pathways with a high \% of reactions showing high correlation with TL. The pathways of glycolysis (A), TCA cycle (B), and lysine degradation to acetyl-CoA (C) are shown. Reactions that have high correlation with TL are represented by solid lines, and the GEM reaction ID is given. Reactions that have low correlation with TL are represented by dotted lines. Key metabolites in each pathway are indicated. F1,6-bP, fructose 1,6-bisphosphate. DHAP, dihydroxyacetone phosphate. G3P, glyceraldehyde 3-phosphate. 1,3-bPG, 1,3-bisphosphoglycerate. 3PG, 3Phosphoglycerate. 2PG, 2-Phosphoglycerate. PEP, phosphoenolpyruvate. OAA, oxaloacetate. CIT, citrate. Suc-CoA, succinyl-CoA. SUC, succinate. FUM, fumarate. MAL, malate.

Figure 4. ATP production is constrained by nucleotide metabolism. $\mathbf{A}$, the nucleotide synthesis pathway from several precursors is shown. Reactions that have high correlation with TL are represented by solid lines. Key metabolites are indicated. Colors separate the main pathway (black) from the synthesis of different precursors. PRPP, phosphoribosyl pyrophosphate. GAR, 5'phosphoribosylglycinamide. N-formyl-GAR, 5'-phosphoribosyl-N-formylglycinamide. AIR, 5'phosphoribosyl-5-aminoimidazole. SAICAR, 1-(5'-phosphoribosyl)-5-amino-4-(Nsuccinocarboxamide)-imidazole. AICAR, 1-(5'-phosphoribosyl)-5-amino-4-(N-succinocarboxamide)imidazole. IMP, inosine monophosphate. 10-formyl-THF, 10-formyltetrahydrofolate. B, the maximum 
bioRxiv preprint doi: https://doi.org/10.1101/2021.07.01.450789; this version posted July 3, 2021. The copyright holder for this preprint (which was not certified by peer review) is the author/funder, who has granted bioRxiv a license to display the preprint in perpetuity. It is made available under aCC-BY-NC-ND 4.0 International license.

411 intake, with $10 \%$ of the food allowed to be adenosine monophosphate (AMP).

412 
Figure 1

A

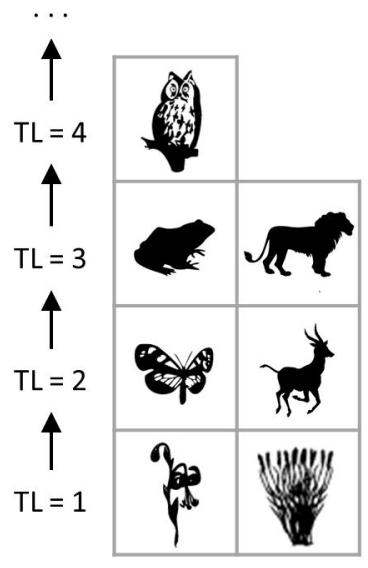

B

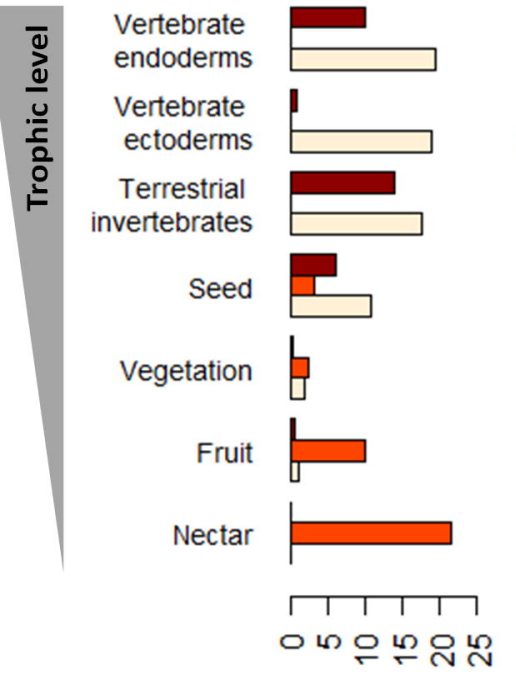

C

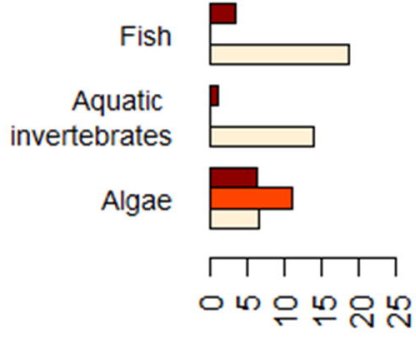

nutrient composition (\%)

nutrient composition (\%)

lipids

carbohydrates

proteins 
Figure 2
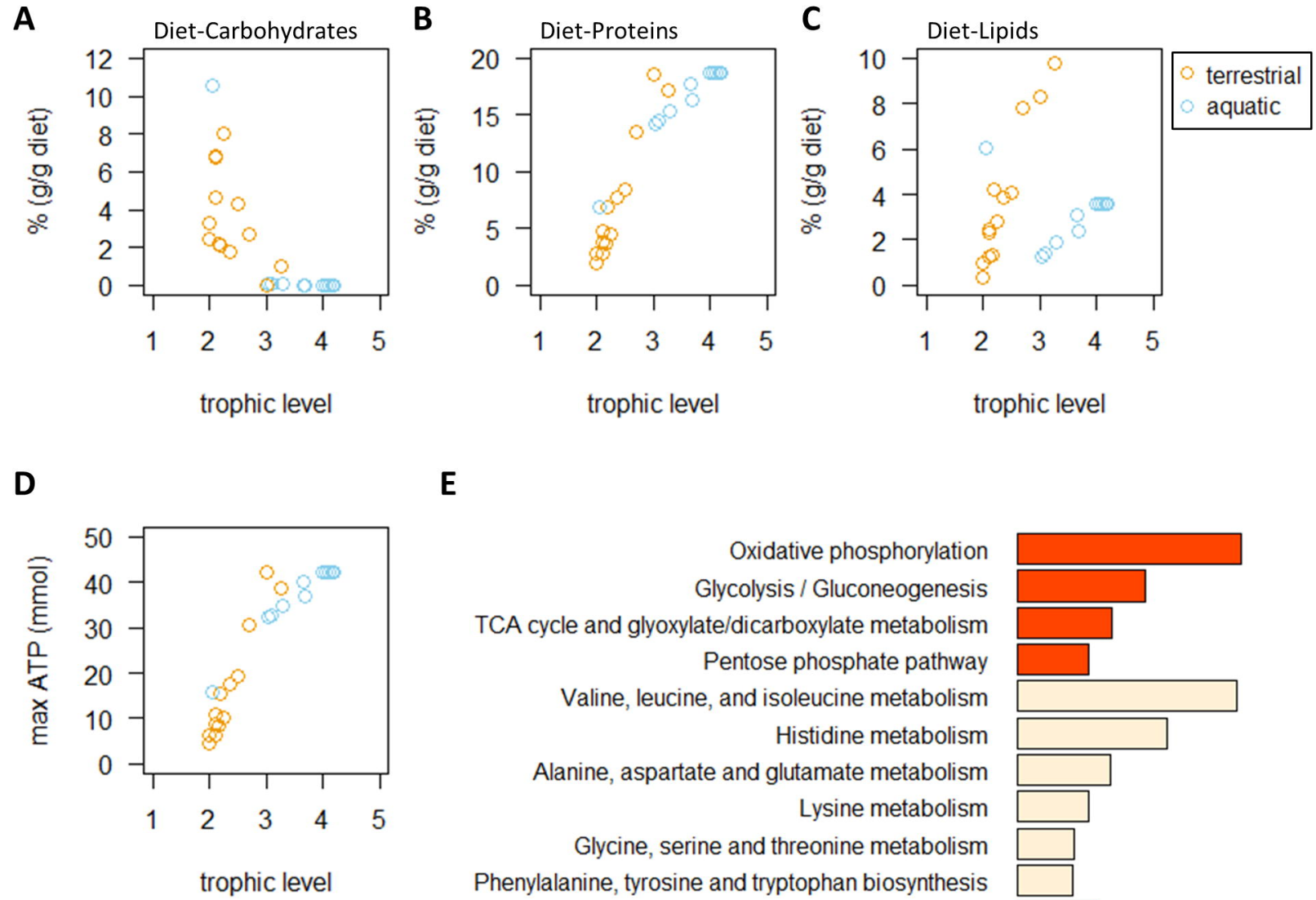

E

Oxidative phosphorylation Glycolysis / Gluconeogenesis TCA cycle and glyoxylate/dicarboxylate metabolism Pentose phosphate pathway Valine, leucine, and isoleucine metabolism Histidine metabolism Alanine, aspartate and glutamate metabolism Lysine metabolism Glycine, serine and threonine metabolism Phenylalanine, tyrosine and tryptophan biosynthesis Fatty acid activation (endoplasmic reticular) Fatty acid oxidation

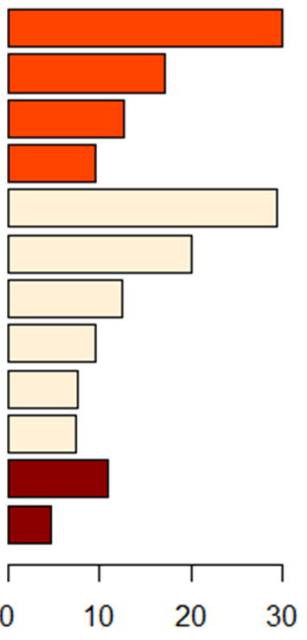

$\%$ rxns correlated with TL 
Figure 3

A
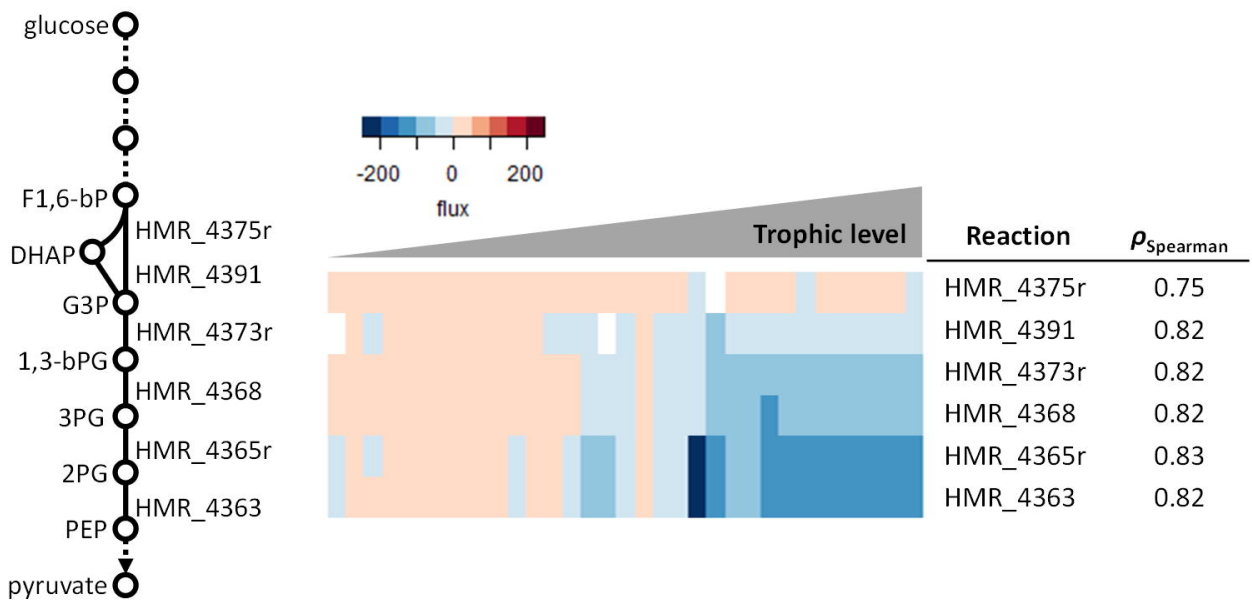

B
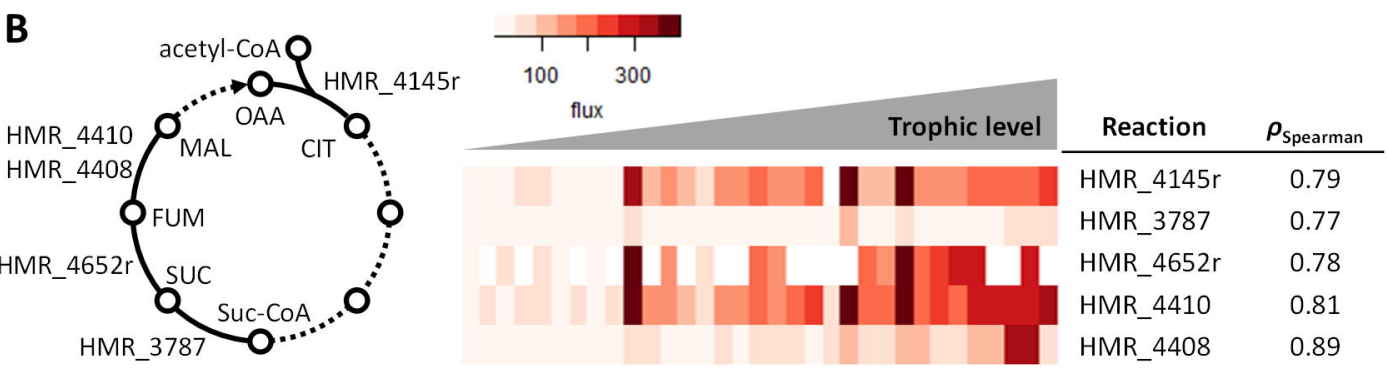

C

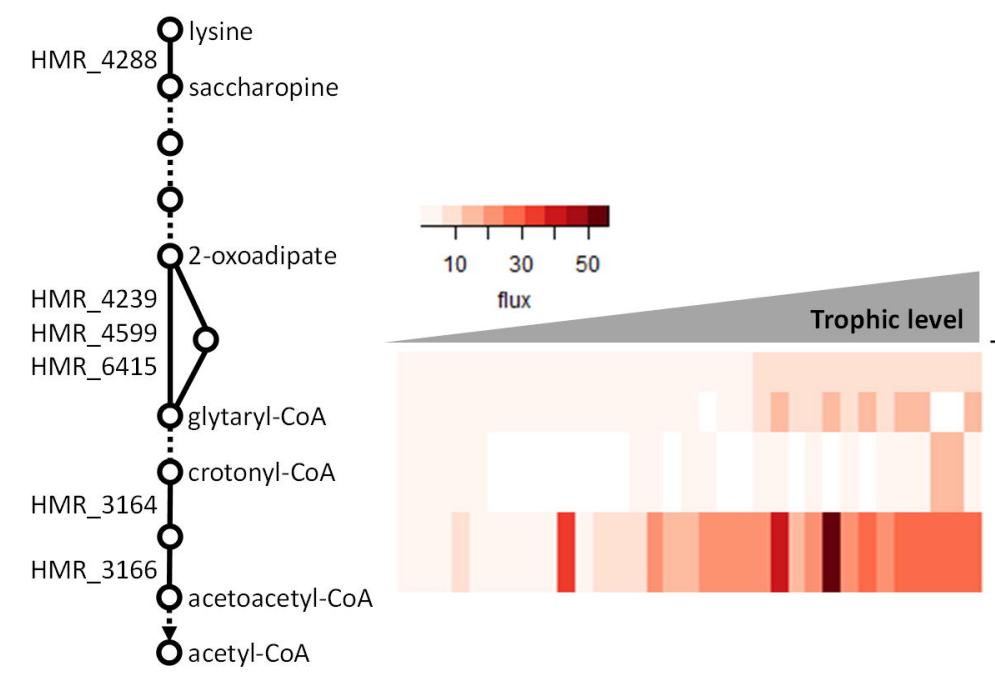

\begin{tabular}{cc} 
Reaction & $\boldsymbol{\rho}_{\text {Spearman }}$ \\
\hline HMR_4288 & 0.94 \\
HMR_4239 & 0.96 \\
HMR_4599 & 0.82 \\
HMR_6415 & 0.82 \\
HMR_3164 & 0.82 \\
HMR_3166 & 0.82
\end{tabular}


Figure 4

A

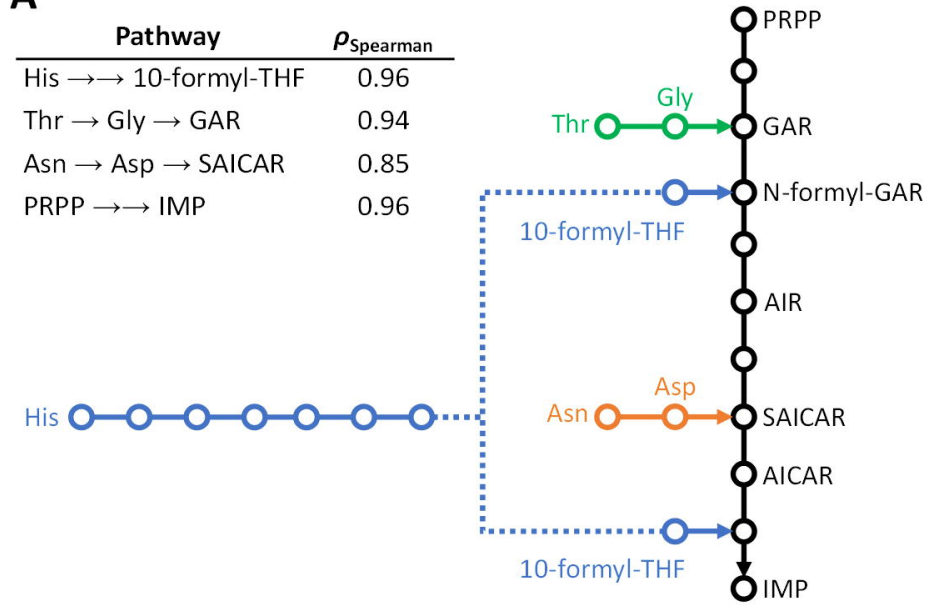

B

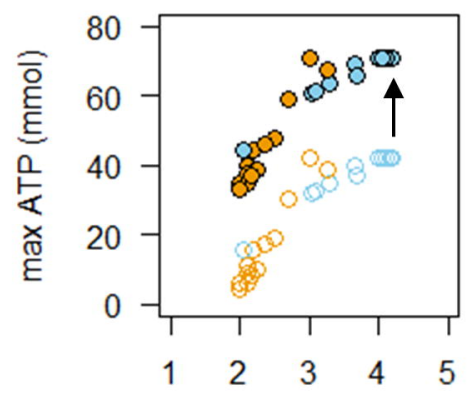

trophic level 